\title{
Tasas diarias de transpiración y relaciones hídricas en especies arbóreas con distinto nivel de sombra tolerancia en un bosque templado chileno
}

\section{Daily transpiration rates and hydraulic relationships in tree species with different shade- tolerance level in a Chilean temperate forest}

\author{
Mylthon Jiménez-Castillo, Paulina Lobos-Catalán, Isabella Aguilera-Betti \& Renato Rivera \\ Laboratorio de Ecología Funcional Vegetal, Facultad de Ciencias, Universidad Austral de Chile, Casilla 567, Valdivia, Chile. \\ mylthonjimenez@uach.cl
}

\begin{abstract}
RESUMEN
En general se sabe que especies pioneras que sostienen altas tasas de crecimiento, transpiración y fotosíntesis son más tolerantes a la sequía y soportan mayores temperaturas, mientras que especies sombra tolerantes lo contrario. Sin embargo, no existen antecedentes respecto a los volúmenes diarios de agua transpirada a nivel de árbol en especies del bosque templado del sur de Chile, ni antecedentes respecto a la coordinación entre los rasgos funcionales involucrados en el transporte de larga distancia. El objetivo de este estudio fue cuantificar los volúmenes diarios de agua transpirada por cuatro especies arbóreas de diferente grado de sombra tolerancia en un bosque templado lluvioso del sur de Chile, así como evaluar su relación con diferentes rasgos funcionales asociados al transporte hídrico en tronco, ramas y hojas. Se encontró que existen diferencias significativas en las tasas diarias de transpiración entre especies, las que varían entre 0,07 y 0,01 lt $\mathrm{cm}^{-2} \mathrm{dia}^{-1}$ para Nothofagus dombeyi (Mirb.) Oerst. y Laureliopsis philippiana (Looser) Schodde respectivamente. Contrario a lo esperado, ni la conductividad hidráulica específica $\left(K_{S}\right)$ ni la conductividad hidráulica foliar $\left(K_{L}\right)$ estuvieron bien correlacionadas con las tasas diarias de transpiración. Sin embargo, las especies menos tolerantes a la sombra presentaron rasgos asociados a una mayor tolerancia a la sequía y mientras que la semi-tolerante Eucryphia cordifolia Cav. estaría sufriendo importantes restricciones hídricas durante el verano.
\end{abstract}

Palabras clave: Transpiración, conductividad hidráulica, transporte de agua.

\begin{abstract}
In general, pioneer species are known for supporting high transpiration, growth and photosynthesis rate, for being more drought tolerant and resist higher temperatures than shade-tolerant species. However, there is not information about rates of transpiration at the tree level in species of Chilean temperate forest, or about the coordination between hydraulic functional traits. We report here, transpiration rates of four tree species with different levels of shade tolerance in Chilean temperate rainforest, and explored some functional traits associated with water transport efficiency and drought tolerance. We found significant differences in daily transpiration rates ranging between 0.07 and $0.01 \mathrm{lcm}^{-2}$ day $^{-1}$ in Nothofagus dombeyi (Mirb.) Oerst. and Laureliopsis philippiana (Looser) Schodde respectively. Contrary to expectations, specific hydraulic conductivity $\left(K_{S}\right)$ and leaf hydraulic conductivity $\left(K_{L}\right)$ were not well correlated with transpiration daily rates. However, the species less tolerant to shade showed traits associated with greater drought tolerance, while the semi-tolerant Eucryphia cordifolia Cav. showed important evidence of hydraulic restrictions during summer.
\end{abstract}

KEYwORDS: Transpiration, hydraulic conductivity, water transport.

\section{INTRODUCCIÓN}

Características ecológicas y fisiológicas determinan el consumo de agua de las especies. Especies pioneras se caracterizan por sostener altas tasas de crecimiento, transpiración y fotosíntesis, son más tolerantes a la sequía y soportan mayores temperaturas, mientras que especies sombra tolerantes lo contrario (Bazzaz 1979, Kitajima 1994, Dalling \& Burslem 2005, Valladares \& Niinemets 2008, Asbjorsen et al. 2011). Al respecto, se ha documentado grandes diferencias en los volúmenes de agua transpirados entre especies. Por ejemplo, Fetene \& Beck 
(2004) encontraron que Croton macrostachys y Eucalyptus globulus transpiran 60 y 551 de agua al día, volúmenes 4 a 5 veces mayores que los transpirados por árboles de Podocarpus falcatus y Cupressus lusitanica, de igual talla y en las mismas condiciones ambientales.

Los volúmenes de agua transpirados $(E)$ y la eficiencia con que ésta es transportada a través de los árboles $\left(K_{S}\right.$ y $K_{L}$ más abajo detallados), están determinados principalmente por la arquitectura hidráulica y disponibilidad de agua en el suelo (McCulloh \& Sperry 2005, Tyree et al. 1991, Patiño et al. 1995, Yang \& Tyree 1994, Calder 1998). Así, las tasas de transpiración a nivel de planta son reflejo de un sistema vascular coordinado e integrado a distintos niveles. El movimiento de agua en las hojas es muy importante ya que la conductancia hidráulica foliar $\left(K_{L}\right)$ explica entre el 30 y $80 \%$ de la resistencia al flujo de agua en la planta (Salleo et al. 1997, Sack et al. 2003), por lo tanto una disfunción del sistema vascular foliar podría tener importantes consecuencias en el transporte hídrico a nivel de planta (Brodribb \& Holbrook 2003, Sack et al. 2003). La conductividad hidráulica específica $\left(K_{S}\right)$ es un indicador de la eficiencia en el transporte hídrico de larga distancia en la planta y suele estar bien correlacionado con $E$ y $K_{L}$ (Brodribb \& Feild 2000, Meinzer 2003, Bond et al. 2007). Rasgos funcionales de la hoja como potencial hídrico foliar $\left(\psi_{\mathrm{L}}\right)$ y punto de perdida de turgor $(P P T)$ están bien correlacionados con el control estomático y transporte en el tallo (Kubiske et al. 1996, Brodribb et al. 2003, Meinzer 2003), siendo además muy buenos indicadores de tolerancia a la sequía (Lambers et al. 1998). Sin embargo, aunque estos rasgos están bien coordinados entre si, existe una gran variabilidad entre especies (Brodribb et al. 2003).

La disponibilidad de agua modula los patrones de diversidad y distribución de plantas, desde microescalas a globales (Field \& Brodribb 2001, Kursar et al. 2009, Bréda et al. 2006), sin embargo, el rol de los rasgos funcionales sobre el desempeño de las plantas bajo restricciones hídricas está pobremente definido (Coomes \& Grubb 2000). El actual escenario de cambio climático, que ha reducido las precipitaciones entre $30 \%$ y $40 \%$ y ha extendido la duración de la estación seca en el centro-sur de Chile (Arnell et al. 2001, Pezoa 2003), podría impactar sobre el rendimiento hidráulico de las especies del bosque, con posibles consecuencias futuras sobre su distribución o la dinámica forestal.

Hasta donde nuestro conocimiento alcanza, no existen antecedentes respecto a las tasas de transpiración global diaria (de toda la planta) para las especies del bosque templado siempreverde del sur de Chile, son pocos los trabajos que abordan la coordinación de rasgos funcionales asociados al transporte de agua (eg. Brodribb et al. 2005, Lusk et al. 2007, Figueroa et al. 2010), y más escasos aún aquellos que abordan su relación con rasgos de historia de vida.
El objetivo de este estudio fue cuantificar los volúmenes diarios de agua transpirada por cuatro especies arbóreas de diferente grado de sombra tolerancia en un bosque templado lluvioso del sur de Chile, así como evaluar su relación con diferentes rasgos funcionales asociados al transporte hídrico en el tronco, ramas y hojas. Dado los antecedentes presentados, se espera que la tasa de transpiración esté inversamente correlacionada con la sombra tolerancia de las especies y positivamente correlacionada con rasgos funcionales asociados a la eficiencia en el transporte hídrico en tronco, ramas y hojas.

\section{METODOLOGÍA}

ÁREA Y ESPECIES DE ESTUDIO

El estudio se realizó en el Bosque Experimental San Martín de la Universidad Austral de Chile, ubicado en la base oriental de la Cordillera de la Costa y colindante con el humedal del Río Cruces ( $39^{\circ} 38^{\prime} \mathrm{S}$ y $73^{\circ} 07^{\prime} \mathrm{W}$; $1-10 \mathrm{~m}$ s.n.m.) en la Región de los Ríos. El periodo de muestreo se realizó al final de la estación seca del año 2009, entre la segunda mitad de febrero y la última semana de marzo. En el área de estudio el clima es templado lluvioso, presentando 2 a 3 meses de sequía estival y una precipitación media anual de $2.240 \mathrm{~mm}$. Las temperaturas medias fluctúan entre $\operatorname{los} 7,3^{\circ} \mathrm{C}$ durante el invierno y $17,5^{\circ} \mathrm{C}$ en verano (di Castri \& Hajek 1976). El suelo es tipo trumao franco-arenoso, con un alto contenido de materia orgánica y de drenaje moderado. La vegetación arbórea es dominada por especies siempreverdes del bosque templado-lluvioso, con presencia de la caducifolia Nothofagus obliqua (Mirb.) Oerst.

Se seleccionaron cuatro especies arbóreas de diferente nivel de sombra tolerancia basados en el estudio de Lusk et al. (2006), donde muestra el nivel de sombra tolerancia de especies arbóreas del bosque templado siempreverde a partir de un análisis cuantitativo del uso del ambiente lumínico. Las especies estudiadas en orden creciente de tolerancia a la sombra corresponden a Nothofagus dombeyi (Nothofagaceae), Eucryphia cordifolia Cav. (Cunoniaceae), Gevuina avellana Molina (Proteaceae) y Laureliopsis philippiana (Looser) Schodde (Atherospermataceae), todas muy abundantes en el sitio de estudio. Todos los individuos seleccionados corresponden a árboles adultos del dosel, con alturas superiores a los $30 \mathrm{~m}$ y diámetros a la altura del pecho (dap) de entre 30 y $50 \mathrm{~cm}$.

\section{TRANSPIRACIÓN}

La tasa de transpiración diaria $\left(E, 1 \mathrm{dia}^{-1}\right)$ fue medida por medio de pruebas de disipación térmica (TDP) de acuerdo a la metodología descrita por Granier (1987). En términos generales, el TDP es un sistema que mide la velocidad de flujo de savia directamente en el xilema, la que luego es convertida a volumen. El sistema consiste en dos sondas 
insertadas en la albura del tronco del árbol, distantes $10 \mathrm{~cm}$ en el eje vertical del árbol y a 1,5 m del suelo. La superior posee una resistencia que produce calor constante mientras que la inferior mide la diferencia de temperatura (dt) entre los dos puntos. Cuando la velocidad del agua es cero dt es máxima. Dado el efecto refrigerante del agua, cuando la velocidad del agua es alta dt es menor. El método de Granier está basado en teoría de velocidad de disipación térmica en líquido y requiere incluir la dimensión física de la madera para convertir velocidad a tasa de flujo:

$$
\mathrm{K}=(\mathrm{dtm}-\mathrm{dt}) / \mathrm{dt} \quad \text { Ecuación } 1
$$

Donde: dt es la diferencia de temperatura entre la resistencia y la aguja ubicada a una distancia fija más abajo, que mide la temperatura ambiente del agua. $\mathrm{dtm}$ es el valor de dt cuando no hay flujo de agua.

Granier (1987) encontró empíricamente que la velocidad promedio del agua en el xilema $\mathrm{V}(\mathrm{cm} / \mathrm{s})$ se relaciona con $\mathrm{K}$ por medio de una expresión exponencial:

$$
\mathrm{V}=0,0119 * \mathrm{~K}^{\wedge} 1,231 \mathrm{~cm} / \mathrm{s}, \quad \text { Ecuación } 2
$$

Al convertir la velocidad en tasa de flujo de agua: $\mathrm{F}_{\mathrm{S}}=$ $\mathrm{A}_{\mathrm{x}} * \mathrm{~V} * 3600(\mathrm{~s} / \mathrm{h}) \mathrm{cm}^{3} / \mathrm{h}$.

Donde $F_{S}$ es el flujo de agua en el xilema $\left(\mathrm{cm}^{3} / \mathrm{h}\right)$, y $A_{x}$ es el área transversal de xilema activo $\left(\mathrm{cm}^{2}\right)$. Obteniendo de esta forma el volumen de agua transpirado.

Se estimó transpiración en al menos cinco individuos por especie, en ciclos continuos de 10 días durante la estación seca. Las mediciones fueron hechas cada $30 \mathrm{seg}$ y se promedió su valor cada $5 \mathrm{~min}$. Estos fueron almacenados en un Data Logger Campbell CR1000.

El área de xilema activo de cada individuo se estimó extrayendo muestras de madera en sentido radial mediante taladros de incremento. La dimensión de la sección hidroactiva se midió identificando sus límites por la diferencia de coloración en la madera al cambiar de albura a duramen.

CONDUCTIVIDAD HIDRÁUliCA ESPEĆ́FICA $\left(K_{S}\right)$

Se midió conductividad hidráulica específica $\left(K_{S}\right)$ como indicador de la eficiencia en el transporte hídrico a nivel de ramas. Para ello se utilizó entre 5 y 7 ramas completamente expuestas, provenientes de al menos 5 individuos diferentes. La conductividad hidráulica específica corresponde a la tasa de flujo de agua movilizado por una fuerza conductora a través de una rama, normalizado por el largo del segmento y referenciado al área transversal de xilema conductor (Ec. 3). Esta medida, al estar normalizada por unidad de área transversal de xilema, hace comparable las mediciones de transporte hídrico entre segmentos de distintos tamaños:

$$
\begin{aligned}
& K_{S}=\mathrm{F} \mathrm{L} /\left(\Delta \mathrm{PA}_{\mathrm{x}}\right) \\
& K_{S}=\mathrm{Kg} \mathrm{s}^{-1} \mathrm{~m}^{-1} \mathrm{MPa}^{-1}
\end{aligned}
$$

Ecuación 3
Donde $\mathrm{F}$ es el flujo de agua movilizado a través del segmento expresado en masa $\left(\mathrm{Kg} \mathrm{s}^{-1}\right)$, $\mathrm{L}$ es el largo del segmento $(\mathrm{m}), \Delta \mathrm{P}$ es la diferencia de presión entre los extremos del segmento $\left(\mathrm{MPa}^{-1}\right), \mathrm{y}_{\mathrm{X}}$ es el área transversal de xilema $\left(\mathrm{m}^{-2}\right)$.

Se midió $K_{S}$ utilizando un sistema de campo para la medición de flujo por caída de presión (Tyree et al. 1993, 1994, Brodribb \& Feild 2000, Zwieniecki et al. 2000). Este sistema consiste en varios microtubos de conductancia hidráulica conocida $\left(\mathrm{k}_{\text {tubo }}=\mathrm{F} / \Delta \mathrm{P}\right)$ y conectados en serie con la muestra. Un reservorio ubicado en altura actúa como cabeza hidráulica, el que por gravedad moviliza el fluido dentro del sistema a presión constante. Un transductor de presión permite conocer la presión con que se moviliza el fluido antes que ingrese al tubo $\left(\mathrm{P}_{1}\right)$ y al salir de este $\left(\mathrm{P}_{2}\right)$, indicando la caída de presión a través del tubo. La caída de presión a través del la muestra corresponde a la diferencia de presión entre los extremos proximal $\left(\mathrm{P}_{2}\right)$ y distal $\left(\mathrm{P}_{3}\right)$ del segmento. Una vez que el flujo se mantiene estable dentro del sistema, este es igual dentro del tubo como en la muestra (Tyree et al. 1993, Brodribb \& Feild 2000). Por lo tanto, $\mathrm{k}_{\text {muestra }}$ puede ser calculado determinando la diferencia de presión a través del tubo calibrado $\left(\Delta \mathrm{P}_{\text {tubo }}\right)$ y la muestra $(\Delta \mathrm{P}$ muestra $)\left(\right.$ Ec. 4). Una vez conocida $\mathrm{k}_{\text {muestra }}$ se calcula $K_{S}$ como se indicó más arriba (Ec. 3), estandarizando por unidad de largo del segmento y área de xilema activo.

$$
\begin{aligned}
& \mathrm{k}_{\text {muestra }}=\mathrm{F} / \Delta \mathrm{P}_{\text {muestra }} \\
& \mathrm{k}_{\text {muestra }}=\Delta \mathrm{P}_{\text {tubo }} \mathrm{k}_{\text {tubo }} / \Delta \mathrm{P}_{\text {muestra }} \quad \text { Ecuación } 4
\end{aligned}
$$

El fluido utilizado corresponde a una solución de KCL $10 \mathrm{mM}$, preparada en agua desionizada y filtrada con una retensión de partícula de $0,2 \mu \mathrm{m}$. Esta solución se utiliza para evitar la reducción de conductividad en la muestra, la que puede ocurrir al utilizar sólo agua pura (Sperry et al. 1987, Zwieniecki et al. 2001).

\section{PotenCIAL HíDRico $(\psi)$}

Se midió el potencial hídrico de medio día $\left(\psi_{\mathrm{md}}\right)$ en hojas completamente expuestas provenientes de entre 5-7 individuos distintos. Para ello se utilizó una bomba de presión (PMS 600, PMS Instruments). El potencial hídrico es un buen indicador del estatus hídrico de la planta y la disponibilidad de agua en el suelo (potencial mátrico).

\section{Punto de PÉRDIDA DE TURGOR}

Mediante curvas presión/volumen se determinó el punto de pérdida de turgor (PPT) de cada especie. Esta es la relación entre el cambio en el contenido relativo de agua de un tejido (RWC) y el inverso del potencial al que se alcanza dicho RWC. El potencial hídrico al que ocurre el PPT se determina por el cambio en la pendiente en la curva. Para la construcción de cada curva se utilizó entre 7 y 10 hojas completamente expuestas provenientes de entre 5 a 6 individuos. 
Conductividad Foliar $\left(K_{L}\right)$

La conductividad foliar indica la capacidad de una hoja para movilizar agua a través de su sistema vascular. Esta es medida por medio del cálculo de la cinética de rehidratación según la metodología descrita por Brodribb \& Holbrook (2003). Una hoja con potencial hídrico conocido $\left(\Psi_{L}\right)$ es puesta a rehidratar a través del pecíolo por un periodo de tiempo fijo. El cambio en el potencial hídrico es usado para calcular conductancia hidráulica foliar. El principio usado es que el mesófilo de la hoja deshidratado actúa como un capacitor que se carga (rehidrata) a través de un resistor, el pecíolo $\left(1 / K_{L}\right)$.

$$
\text { Luego } K_{L}=\mathrm{C} \ln \left(\Psi_{\mathrm{o}} / \Psi_{\mathrm{f}}\right) / \mathrm{t}
$$

Donde C es la capacitancia de la hoja; $\Psi_{\mathrm{o}}$ es el potencial hídrico antes de la rehidratación; $\Psi_{\mathrm{f}}$ es el potencial hídrico después de la rehidratación, por t segundos. La capacitancia de la hoja se obtiene por medio de curvas presión volumen. La relación entre $\Psi_{\mathrm{L}} \mathrm{y}$ el contenido relativo de agua en la hoja se obtiene utilizando la técnica de secado y peso (Tyree \& Hammel 1972). Los potenciales hídricos fueron medidos con una cámara de presión (Cámara de Scholander).

\section{CuRVAS DE VULNERABILIDAD FOLIAR}

La curva de vulnerabilidad indica cómo varía la capacidad de conducir agua en la hoja a distintos niveles de estrés hídrico, medido como potencial hídrico ( $\Psi)$. De acuerdo a la metodología descrita por Brodribb \& Holbrook (2004), entre 15 y 20 hojas son extraídas y puestas a desecar a temperatura ambiente por distintos periodos de tiempo. En seguida, las hojas son puestas en bolsas plásticas, en oscuridad, por 30 min para evitar que se sigan deshidratando y producir el cierre de los estomas. Luego $K_{L}$ es medido como se indicó más arriba y es graficada contra su potencial hídrico inicial. La curva de vulnerabilidad permite conocer los niveles de tolerancia al estrés hídrico de cada especie y como afecta su capacidad de conducir agua.

\section{ANÁLISIS ESTADÍSTICO}

El efecto de las especies sobre transpiración $(E)$, conductividad hidráulica específica $\left(K_{S}\right)$ y la relación área basal/xilema activo $\left(\mathrm{A}_{\mathrm{x}} / \mathrm{A}_{\mathrm{B}}\right)$ fue analizado por medio de ANOVA y posterior test de Tukey, utilizando el Software JMP (SAS Institute, Cary, NC).

\section{RESULTADOS}

Las tasas de transpiración fueron significativamente diferentes entre especies (Fig. 1). La pionera Nothofagus dombeyi sostuvo las más altas tasas de transpiración correspondientes a un volumen diario de $891 \mathrm{dia}^{-1}$, mientras que la tolerante L. philippiana transpiró en promedio 3,8 $1 \mathrm{dia}^{-1}$ (Tabla I). Para eliminar el efecto de las diferencias de tamaño entre individuos, $E$ fue estandarizado por el área de xilema activo, lo que arrojó tasas de transpiración $\left(E_{s t}\right)$ equivalentes a $0,071 \mathrm{~cm}^{-2} \mathrm{dia}^{-1}$ y $0,01 \quad 1 \mathrm{~cm}^{-2} \mathrm{dia}^{-1}$ respectivamente. Contrario a lo esperado, no se encontró una relación positiva entre las tasas de transpiración y la eficiencia de transporte hídrico en ramas ni en hojas.

La especie semi tolerante Gevuina avellana mostró la mayor eficiencia de transporte hídrico en ramas $\left(K_{S}\right)$ y hojas

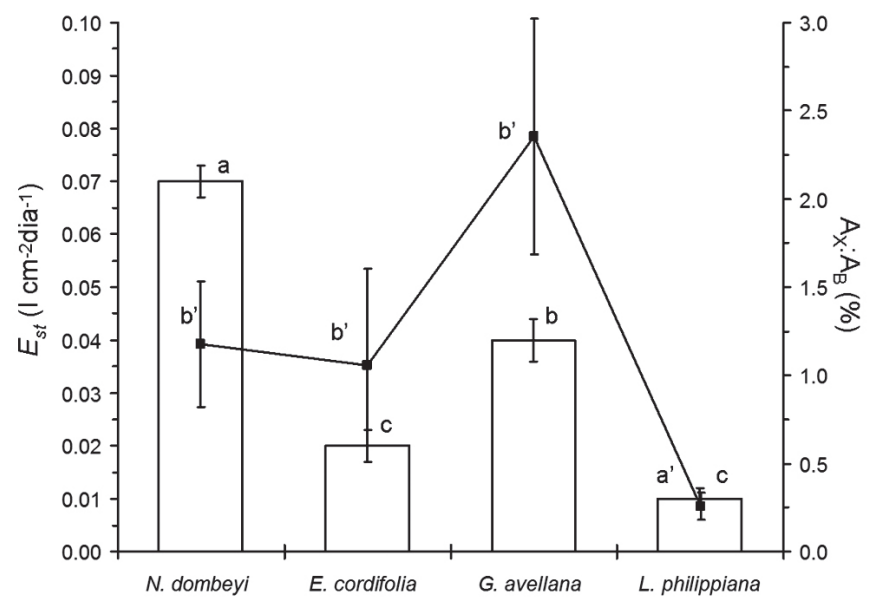

Figura 1. Tasa de transpiración promedio diario $E_{s t}\left(\mathrm{~cm}^{-2} \mathrm{dia}^{-1}\right)$ y proporción de área xilema activo en área basal. Anova para $E_{s t}, F(3,18)$ $10,881 p<0,0001$ (a) N. dombeyi difiere de las otras especies (Tukey $p<0,0001 ; 0,006 ; 0,0001$ ) respectivamente. (b) $G$. avellana difiere de $L$. philippiana (Tukey, $p=0,014$ ). Anova para $\mathrm{A}_{\mathrm{X}} / \mathrm{A}_{\mathrm{B}}, F(3,13) 4,31 p<0,025$ (a) L. philippiana difiere de las otras especies (Tukey, $p<0,05$ ).

Figure 1. Mean daily of transpiration rate $E_{s t}\left(\mathrm{lcm}^{-2} \mathrm{dia}^{-1}\right)$ and ratio of active xylem area on basal area. $F(3,18) 10,881 p<0,0001$ (a) $N$. dombeyi is different to other species (Tukey, $p<0,0001 ; 0,006 ; 0,0001$ ) respectively. (b) G. avellana is different to L. philippiana (Tukey, $p=0,014)$. Anova para $\mathrm{A}_{\mathrm{X}} / \mathrm{A}_{\mathrm{B}}, F(3,13) 4,31 p<0,025$ (a) L. philippiana is different to other species (Tukey, $\left.p<0,05\right)$. 
$\left(K_{L}\right)$, así como una mayor proporción de xilema activo por unidad de área basal, sin embargo esto no se tradujo en altas tasas de transpiración (Tabla I, Fig. 1).

Las curvas de vulnerabilidad mostraron que las especies estudiadas sufren un $50 \%$ de pérdida de $K_{L}$ a potenciales cercanos a $1 \mathrm{Mpa}$. Sin embargo, la pérdida de conductividad hidráulica foliar a $\psi_{\text {md }}$ ocurre en un rango de entre $63 \%(N$. dombeyi) a 50\% (G. avellana) como consecuencia del estrés hídrico (Fig. 2).
Se encontró que la pionera $N$. dombeyi es la especie con mayor tolerancia al estrés hídrico, ya que mostró un punto de pérdida de turgor de -2Mpa, casi dos veces superior a las otras especies (Fig. 2). Por el contrario, Eucryphia cordifolia mostró una alta vulnerabilidad al estrés hídrico al presentar un $\psi_{\text {md }}$ más negativo que su punto de pérdida de turgor (-1,4Mpa v/s -1.2Mpa) (Fig. 2). Esto podría redundar en una serie de restricciones en productividad y desempeño durante la estación de crecimiento.

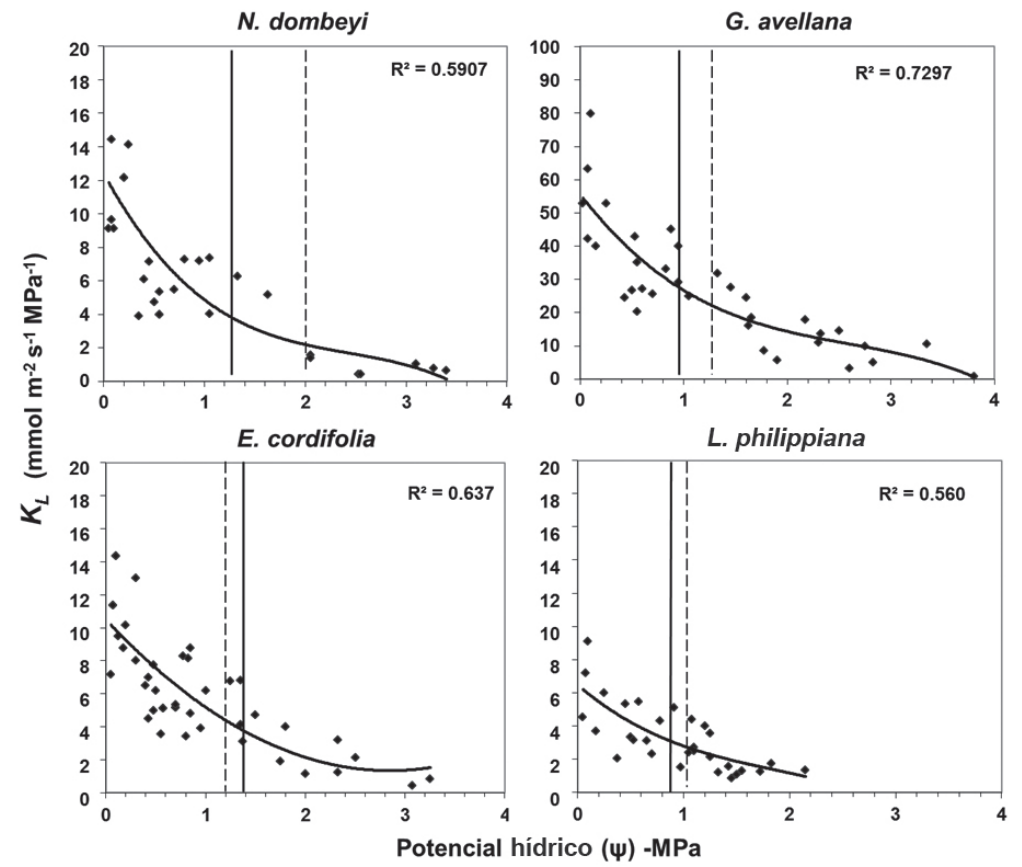

Figura 2. Curvas de vulnerabilidad de hoja para las cuatro especies en estudio. Línea continua muestra el potencial hídrico de mediodía $\left(\Psi_{\mathrm{md}}\right)$ y la línea segmentada el punto de pérdida de turgor (PPT).

Figure 2. Leaf vulnerability curves for species in study. Continuous line represent mid day water potential $\left(\Psi_{\text {md }}\right)$ and doted line show the turgor lost point (PPT).

TABLA I. Valores promedio por especie y error estándar para las tasas netas de transpiración diaria $(E)($ Anova $F(3,18) 19,46 p<0.0001)$, tasas de transpiración estandarizadas $\left(E_{s t}\right)$ (Anova $\left.F(3,18) 10,881 p<0,0001\right)$, conductividad hidráulica específica $\left(K_{s}\right)$ (Anova $F(3$, 18) $14,12 p<0,0004)$ y proporción de xilema activo en el área basal $\left(\mathrm{A}_{\mathrm{x}} / \mathrm{A}_{\mathrm{B}}\right)$ (Anova $\left.F(3,18) 4,31 p<0,025\right)$. Letras diferentes indican diferencias significativas entre las especies (Tukey HSD, $p<0,05)$. Conductividad hidráulica foliar $\left(K_{L}\right)$ estimada a partir de curvas de vulnerabilidad.

TABLE I. Mean values and standard error for daily transpiration net rate $(E)$ (Anova $F(3,18) 19.46 p<0.0001$ ), standardized transpiration rate $\left(E_{s t}\right)$ (Anova $F(3,18) 10.881 p<0.0001$ ), specific hydraulic conductivity $\left(K_{s}\right)$ (Anova $F(3,18) 14.12 p<0.0004$ ) and ratio of active xylem area on basal area $\left(\mathrm{A}_{\mathrm{x}} / \mathrm{A}_{\mathrm{B}}\right)$ (Anova $\left.F(3,18) 4.31 p<0.025\right)$. Different letters show significant differences among species $($ Tukey HSD, $p<0.05)$. Leaf hydraulic conductivity $\left(K_{L}\right)$ estimated from vulnerability curves.

\begin{tabular}{|c|c|c|c|c|c|}
\hline Especies & $\begin{array}{c}E \\
\left(1 \mathrm{dia}^{-1}\right)\end{array}$ & $\begin{array}{c}E_{s t} \\
\left(1 \mathrm{~cm}^{-2} \mathrm{dia}^{-1}\right)\end{array}$ & $\begin{array}{c}K_{L} \\
\left(\mathrm{mmol} \mathrm{s}^{-1} \mathrm{Mpa}^{-1} \mathrm{~m}^{-1}\right)\end{array}$ & $\begin{array}{c}K_{s} \\
\left(\mathrm{~kg} \mathrm{~s}^{-1} \mathrm{Mpa}^{-1} \mathrm{~m}^{-1}\right)\end{array}$ & $\mathrm{A}_{\mathrm{x}} / \mathrm{A}_{\mathrm{B}}(\%)$ \\
\hline N. dombeyi & $89,6 \pm 14,1^{a}$ & $0,07 \pm 0,003^{a}$ & 12 & $0,73 \pm 0,07^{b}$ & $1,17 \pm 0,3^{b}$ \\
\hline E. cordifolia & $15,9 \pm 2,75^{c}$ & $0,02 \pm 0,003^{c}$ & 10 & $0,94 \pm 0,14^{b}$ & $1,05 \pm 0,5^{b}$ \\
\hline G. avellana & $12,6 \pm 1,20^{b}$ & $0,04 \pm 0,004^{b}$ & 55 & $3,33 \pm 0,51^{a}$ & $2,35 \pm 0,6^{b}$ \\
\hline L. philippiana & $3,8 \pm 0,75^{c}$ & $0,01 \pm 0,002^{c}$ & 6 & $0,68 \pm 0,05^{b}$ & $0,25 \pm 0,07^{a}$ \\
\hline
\end{tabular}




\section{DISCUSIÓN}

Las especies estudiadas mostraron una gran variación en el consumo neto de agua según su nivel de sombra-tolerancia. Esta variación alcanzó diferencias de más de 20 veces en el volumen neto transpirado (Tabla I), y en 7 veces el volumen estandarizado, entre la pionera Nothofagus dombeyi y la especie de mayor sombra tolerancia Laureliopsis philippiana (Tabla I, Fig. 1). Es conocido que las especies pioneras mantienen altas tasas de transpiración, necesarias para sostener altas tasas de crecimiento (Arkley 1963, Bazzaz 1979, Lambers et al. 1998, Asbjornsen et al. 2011). Por ejemplo, Moore et al. (2004) reportó que especies de coníferas del hemisferio norte, sucesionalmente tardías, transpiran hasta un $30 \%$ menos que las pioneras. Sin embargo, la magnitud de las diferencias en transpiración no había sido documentada para árboles chilenos. Por otra parte, la posición de las especies en el estrato vertical del bosque también puede tener una importante relación con su tasa de transpiración, ya que se ha documentado que especies que poseen una posición emergente pueden transpirar hasta 10 veces más que especies del dosel inferior (Motzer et al. 2005), como en el caso de $N$. dombeyi.

Respecto a la eficiencia en el transporte $\left(K_{S}\right.$ y $\left.K_{L}\right)$, varios trabajos han mostrado una correlación entre $E$ y otros rasgos que determinan la eficiencia en transporte hídrico (Kubiske et al. 1996, Brodribb et al. 2003, Sack et al. 2003, Bucci et al. 2004, Sack \& Tyree 2005, Bond et al. 2007, Asbjornsen et al. 2011). Plantas con alta tasa de transpiración suelen tener altas $K_{L}, K_{S}$ y altas relaciones de $\mathrm{A}_{\mathrm{x}} / \mathrm{A}_{\mathrm{B}}$, mientras que plantas con tasa de transpiración menores lo contrario (Brodribb \& Feild 2000, Meinzer 2003, Brodribb et al. 2003, Poorter et al. 2010). Sin embargo, nosotros no encontramos una clara relación de $E$ (ni de $E_{s t}$ ) con los rasgos estudiados, probablemente debido a que estos rasgos también pueden alcanzar alta variabilidad interespecífica haciendo dificultoso capturar las relaciones entre sólo 4 especies, sobre todo en ambientes naturales donde las especies poseen diferentes rasgos de historia de vida (Bond et al. 2007). En estos sistemas, los individuos ocupan diferentes posiciones en el dosel, o sufren mayor o menor competencia con el vecino, lo que puede tener un efecto sobre variables como el índice de área foliar (LAI) que varía producto de la competencia por luz, o con la edad del árbol (Bond et al. 2007, Asbjornsen et al. 2011), y que a su vez puede influenciar fuertemente las tasas de transpiración (Kitajima 1994, Meinzer 2003, Valladares \& Niinemets 2008, Bartolo et al. 2010).

La especie pionera $N$. dombeyi mostró una mayor tolerancia a la sequía respecto a especies más sombra tolerantes. Esto es evidenciado al presentar un punto de pérdida de turgor (PPT) casi dos veces mayor a las demás especies (Fig. 2). Dado que PPT está fuertemente coordinado con el control estomático, es decir las plantas cierran sus estomas a potenciales justo por delante del punto de pérdida de turgor (Brodribb et al. 2003, Brodribb \& Holbrook 2004), N. dombeyi sería capaz de mantener sus estomas abiertos y seguir transpirando a potenciales más negativos que las otras especies estudiadas. De hecho, su $\Psi_{\text {md }}$ es mayor que en las demás especies (Fig. 2), indicando que podría sufrir importantes niveles de estrés hídrico y aún así seguir realizando intercambio de gases. Esto le permitiría fotosintetizar a potenciales hídricos foliares en que otras especies han cerrado sus estomas, y con ello ser más eficientes en fijar carbono (Brodribb \& Holbrook 2004, Sack et al. 2003, Kursar et al. 2009), sustentando así las altas tasas de crecimiento que le son características (Lusk $\&$ del Pozo 2002).

Por el contrario, Eucryphia cordifolia evidencia restricciones hídricas asociadas a la sequía estival al presentar $\Psi_{\text {md }}$ día más negativos que su PPT (Fig. 2). Debido a que los estomas estarían cerrados a este potencial (Brodribb et al. 2003, Brodribb \& Holbrook 2004) y a la fuerte coordinación entre $K_{L}$, tasa fotosintética $\left(A_{\max }\right)$ y $G_{s}$ (Brodribb \& Feild 2000, Meinzer 2003, Brodribb et al. 2003, Sack et al. 2003, Kursar et al. 2009) este resultado sugiere una restricción de la capacidad fotosintética de la planta al reducir el intercambio gaseoso por estrés hídrico. Cabe destacar que tanto $\Psi_{\mathrm{md}}$ como PPT están dentro del rango de valores documentados para E. cordifolia en el sur de Chile. Por ejemplo, Figueroa et al. (2010) registraron valores de $\Psi_{\mathrm{md}}$ y PPT de 1,15 Mpa y 1,4 MPa respectivamente para el sitio de Cordillera Pelada, cercano a nuestra área de estudio y con un régimen de precipitaciones de similar magnitud (2.363mm/año). Por lo anterior, probablemente nosotros registramos un $\Psi_{\mathrm{md}}$ más negativo que el PPT, debido a que nuestro estudio, a diferencia del de Figueroa et al. (2010), se efectuó al final de la temporada estival pudiendo capturar una situación particular de estrés hídrico en la población local.

En un contexto de cambio climático, en el que las precipitaciones se han reducido entre un 30 y $40 \%$ en el centro sur de Chile (Arnell et al. 2001) y se proyecta una reducción aún mayor, E. cordifolia podría ser mucho más vulnerable a la sequía que otras especies, pudiendo generar cambios en su límite de distribución y/o en la estructura del bosque. Esto, ya que existe evidencia que las poblaciones de E. cordifolia están adaptadas localmente a las condiciones de humedad, disminuyendo su tolerancia al estrés hídrico (eg. punto pérdida turgor) a mayores latitudes (Figueroa et al. 2010). Dado que los individuos estudiados por nosotros han sufrido un proceso de adaptación local a condiciones de mayor humedad que las actuales, es posible suponer que esta población se está enfrentando a situaciones cercanas a su límite de tolerancia al estrés hídrico, o al menos a eventos puntuales, como lo sugiere nuestro resultado. Por supuesto es necesario realizar estudios directos respecto al impacto de la reducción de precipitaciones sobre la adecuación biológica de esta especie para confirmar esta idea.

En resumen, los volúmenes de agua transpirados por 
especies arbóreas pioneras son mucho mayores que los transpirados por especies tolerantes, sin embargo, éstos no parecen estar coordinados con la eficiencia en el transporte hídrico de ramas $\left(K_{S}\right)$ ni hojas $\left(K_{L}\right)$. Por otra parte, se evidencia que la especie $E$. cordifolia se enfrenta a una condición de restricción hídrica, que hace suponer una baja capacidad de tolerar sequías prolongadas, augurando una reducción de su adecuación biológica en un escenario de disminución de precipitaciones.

\section{AGRADECIMIENTOS}

Este estudio fue financiado por CONICYT a través del proyecto FONDECYT 11060404. Agradecemos a quienes desinteresadamente colaboraron en el trabajo de campo, especialmente a Eniuska Azócar y Prisilla Erber por su buena disposición. Consideración especial a don Pedro quien siempre colaboró con el equipo de trabajo y facilitó nuestra labor en la Estación Experimental San Martín.

\section{BIBLIOGRAFÍA}

Arkley, R. 1963. Relationships between plant growth and transpiration. Hilgardia 34(13): 559-584.

Arnell, N., C. Liu, R. Compagnucci, L. da Cunha, K. Hanaki, C. Howe, G. Mailu, I. Shiklomanov \& E. Stakhiv. 2001. Hydrology and Water Resources. In: J.J. McCarthy, O.F. Canziani, N.A. Leary, D.J. Dokken \& K.S. White (eds.), Climate Change 2001. Impacts, Adaptation, and Vulnerability. pp.191-233. World Meteorological Organisation/United Nations Environment Programme. Cambridge University Press, Australia.

Asbjornsen, H., G.R. Goldsmith, M.S. Alvarado-Barrientos, K. Rebel, F.P. Van Osch, M. Rietkerk, J. Chen, S. Gotsch, C. Tobón, D.R. Geissert, A. Gómez-Tagle, K. Vache \& T.D. DAwson. 2011. Ecohydrological advances and applications in plant-water relations research: a review. Journal of Plant Ecology 4(1-2): 3-22.

Bartolo, C., C.E.T. Paine, L. Poorter, J. Beauchene, D. Bonal, A.M. Domenach, B. Hérault, S. Patiño, J.C. Roggy \& J. Chave. 2010. Decoupled leaf and stem economy in rain forest trees. Ecology Letters 13(11): 1338-1347.

BAzZAZ, F.A. 1979. The physiological ecology of plant succession. Annual Review of Ecology and Systematics 10(1): 351371.

Bond, B.J, F.C. Meinzer \& J.R. BRoOKs. 2007. How Trees Influence the Hydrological Cycle in Forest Ecosystems. In: P.J. Wood, D.M. Hannah \& J.P. Sadler (eds.), Hydroecology and Ecohydrology: Past, Present, and Future. Pp. 7-36. Willey.

Bréda, N., R. Huc, A. Granier \& E. Dreyer. 2006. Temperate forest trees and stands under severe drought: a review of ecophysiological responses, adaptation processes and long-term consequences. Annals of Forest Science 63(6): 625-644.
Brodribb, T.J. \& T.S. Feild. 2000. Stem hydraulic supply is linked to leaf photosynthetic capacity: evidence from New Caledonian and Tasmanian rainforest. Plant, Cell, and Environment 23(12): 1381-1388.

BrodribB, T.J. \& N.M. Holbrook. 2003. Stomatal closure during leaf dehydration, correlation with other leaf physiological traits. Plant Physiology 132(4): 2166-2173.

Brodribb, T.J., N.M. Holbrook, E.J. Edwards \& M.V. Gutiérrez. 2003. Relations between stomatal closure, leaf turgor and xylem vulnerability in eight tropical dry forest trees. Plant, Cell \& Environment 26(3): 443-450.

Brodribb, T.J. \& N.M. Holbrook. 2004. Diurnal depression of leaf hydraulic conductance in a tropical tree species. Plant, Cell and Environment 27: 820-827.

Brodribb, T.J., N.M. Holbrook, M.A. Zwieniecki \& B.Palma. 2005. Leaf hydraulic capacity in ferns, conifers and angiosperms: impacts on photosynthetic maxima. New Phytologist 165: 839-846.

Bucci, S., G. Goldstein, F.C. Meinzer, F.G. Scholz, A.C. Franco \& M. Bustamante. 2004. Functional convergence in hydraulic architecture and water relations of tropical savanna trees: from leaf to whole plant. Tree Physiology 24: 891-899.

CAlder, I.R. 1998. Water use by forests, limits and controls. Tree Physiology 18: 625-631.

Coomes, D.A. \& P.J. GrubB. 2000. Impacts of root competition in forests and woodlands: A theoretical framework and review of experiments. Ecological Monograph 70: 171-207.

DALLING, J.W. \& D.F.R.P. BursLEM. 2005. Role of life-history tradeoffs in the equalization and differentiation of tropical tree species. In: D.F.R.P. Burslem, M.A. Pinard \& S.E. Hartley (eds.), Biotic interactions in the tropics: Their role in the maintenance of species diversity, pp. 65-88. Cambridge University Press, Cambridge, UK.

Di Castri, F. \& E. Hajek. 1976. Bioclimatología de Chile. Editorial Universidad Católica de Chile, Santiago. 129 pp.

Fetene, M. \& E.H. BeCK. 2004. Water relations of indigenous versus exotic tree species, growing at the same site in a tropical montane forest in southern Ethiopia. Trees 18: 428-435.

Field, T. \& T. BRodribB. 2001. Stem water transport and freezethaw xylem embolism in conifers and angiosperms in a Tasmanian treeline heath. Oecologia 127: 314-320.

Figueroa, J.A., H.M. Cabrera, C. Queirolo \& L.F. Hinojosa. 2010. Variability of water relations and photosynthesis in Eucryphia cordifolia Cav. (Cunoniaceae) over the range of its latitudinal and altitudinal distribution in Chile. Tree physiology 30: 574-585.

Granier, A. 1987. Evaluation of transpiration in a Douglas-fir stand by means of sap flow measurements. Tree Physiology 3: 309-320.

Kiталাма, K. 1994. Relative importance of photosynthetic and allocation traits as correlates of seedling shade tolerance of 15 tropical tree species. Oecologia 98(3-4): 419-428.

Kubiske, M.E., M.D. Abrams \& S.A. Mostoller. 1996. Stomatal and nonstomatal limitations of photosynthesis in relation to the drought and shade tolerance of tree species in open and understory environments. Trees 11(2): 76-82.

Kursar, T.A., B.M.J. Engelbrecht, A. Burke, M.T. Tyree, B.E. Omari \& J.P. GIRALDO. 2009. Tolerance to low leaf water status of 
tropical tree seedlings is related to drought performance and distribution. Functional Ecology 23: 93-102.

Lambers, H., F.S. Chapin \& T. Pons. 1998. Plant physiological ecology. Springer-Verlag, New York, USA. 540 pp.

Lusk, C.H. \& A.DEL Pozo. 2002. Survival and growth of seedlings of 12 chilean rainforest trees in two light environments: gas exchange and biomass distribution correlates. Austral Ecology 27: 173-182.

Lusk, C.H., R.L. Chazdon \& G. Hofmann. 2006. A bounded null model explains juvenile tree community structure along light availability gradients in a temperate rain forest. Oikos 112: 131-137.

Lusk, C.H., M. Jiménez-Castillo \& N.Salazar-Ortega. 2007. Evidences that branches of evergreen angiosperms and coniferous trees differ in hydraulic conductance but not in Huber values. Canadian Journal of Botany 85: 141-147.

McCulloh, K. \& J. Sperry. 2005. Patterns in hydraulic architecture and their implications for transport efficiency. Tree Physiology 25(3): 257-267.

MeinZer, F. 2003. Functional convergence in plant responses to the environment. Oecologia 134(1): 1-11.

Moore, G.W., B.J. Bond, J.A. Jones, N. Phillips \& F.C. Meinzer. 2004. Structural and compositional controls on transpiration in a 40- and 450-yr-old riparian forest in western Oregon, USA. Tree Physiology 24: 481-4.

Motzer, T., N. Munz, M. Küppers, D.Schmitt \& D.Anhuf. 2005. Stomatal conductance, transpiration and sap flow of tropical montane rain forest trees in the southern Ecuadorian Andes. Tree Physiology 25: 1283-1293.

Patiño, S., M.T. Tyree \& E.A. Herre. 1995. Comparison of hydraulic architecture of woody plants of differing phylogeny and growth form with special reference to free standing and hemi-epiphytic Ficus species from Panama. New Phytologyst 129(1): 125-134.

PezoA, L. 2003. Recopilación y análisis de la variación de las temperaturas (período 1965-2001) y las precipitaciones (período 1931-2001) a partir de las estaciones meteorológicas entre $\operatorname{los} 33^{\circ}$ y $53^{\circ}$ de latitud sur. Tesis Ing. Forestal, Universidad Austral de Chile, Chile.

Poorter, L., I. McDonald, A. Alarcón, E. Fichtler, J.C. Licona, M. Peña-Claros, F. Sterck, Z. Villegas \& U. SassKLAASSEN. 2010. The importance of wood traits and hydraulic conductance for the performance and life history strategies of 42 rainforest tree species. New Phytologist 185: 481-492.

Sack, L., P.D. Cowan, N. Jaikumar \& N.M. Holbrook. 2003. The 'hydrology' of leaves: co-ordination of structure and function in temperate woody species. Plant, Cell and Environment 26: 1343-1356.

SACK, L. \& M.T. TYreE. 2005. Leaf Hydraulics and its Implications in Plant Structure and Function. In: N.M. Holbrook \& M.A. Zwieniecki (eds.), Vascular Transport in Plants, pp. 93-114. Elsevier (Academic Press).

SAlleo, S., A.NARdini \& M. Lo Gullo. 1997. Is sclerophylly of Mediterranean evergreens an adaptation to drought?. New Phytologist 135(4): 606-312.

Sperry, J.S., M.N. Holbrook, M.H. Zimmermann \& M.T. Tyree. 1987. Spring filling of xylem vessels in wild grapevine. Plant Physiology 83(2): 414-417.

Tyree, M.T. \& H.T. Hammel. 1972. The measurement of the turgor pressure and the water relations of plants by the pressurebomb technique. Journal of Experimental Botany 23(1): 267-282.

Tyree, M.T., D.A. Snyderman, T.R. Wilmot \& J.L. Machado. 1991. Water relations and hydraulic architecture of a tropical tree (Schefflera morototoni). Data, models, and a compararison with two temperate species (Acer saccharum and Thuja occidentalis). Plant Physiology 96(1): 1105-1113.

Tyree, M.T., B. Sinclair, P. Lu \& A.Granier. 1993. Whole shoot hydraulic resistance in Quercus species measured with a new high-pressure flowmeter. Annals of Forest Science 50(5): 417-423.

Tyree, M.T., S. Yang, P.Cruiziat \& B. Sinclair. 1994. Novel methods of measuring hydraulic conductivity of tree root system and interpretation using AMAIZED (A maizeroot dynamic model for water and solute transport). Plant Physiology 104(1): 189-199.

Valladares, F. \& U. NiINEMETs. 2008. Shade tolerance, a key plant feature of complex nature and consequences. The Annual Review of Ecology, Evolution and Systematics 39: 23757.

YAnG, S. \& M.T. Tyree. 1994. Hydraulic architecture of Acer saccharum and A. rubrum: comparison of branches to whole trees and the contribution of leaves to hydraulic resistance. Journal of Experimental Botany 45(2): 179186.

Zwieniecki, M.A., L. Hutyra, M.V. Thompson \& N.M. Holbrook. 2000. Dynamic changes in petiole specific conductivity in red maple (Acer rubrum L.), tulip tree (Liriodendron tulipifera L.), and northern foxgrape (Vitis labrusca L.). Plant, Cell and Environment 23(4): 407-414.

Zwieniecki, M.A., P.J. Melcher \& N.M. HolbrooK. 2001. Hydrogel control of xylem hydraulic resistance in plants. Science 291: 1059-1062.

Recibido: 18.04.11

Aceptado: 11.07.11 